\title{
Oncoradiology Preparedness in the COVID-19 Pandemic: Perspective from a Tertiary Oncology Referral Center from Eastern India
}

\begin{abstract}
At the time of writing this article, more than 18 million people worldwide have been infected by the severe acute respiratory syndrome-associated coronavirus- 2 and about 700,000 people have died from coronavirus disease 2019 (COVID-19). In India, about 190,000 people have been infected and nearly 39,000 people have succumbed to this infection. Infection among health-care workers has emerged as one of the key problems in facing this pandemic. The purpose of this article is to describe the measures taken by the department of oncoradiology at our institution to control the infection and minimize staff exposure during the current lockdown period with reduced patient load and in the post-lockdown period with increased demand for radiology services. The key focus of this article is the continued delivery of cancer imaging services with practical precautions and optimized resources. We have also discussed algorithms and protocols unique to the practice of oncoradiology in the time of the COVID-19 pandemic.
\end{abstract}

Keywords: Coronavirus disease 2019, oncoradiology, personal protection, preparedness

\section{Introduction}

At the time of writing this article, more than 18 million people worldwide have been infected by the severe acute respiratory syndrome-associated coronavirus-2 (SARSCoV-2) and about 700,000 people have died from coronavirus disease 2019 (COVID-19). In India, about 190,000 people have been infected and nearly 39,000 people have succumbed to this infection. Our hospital is in the eastern outskirts of Kolkata in a red zone or "hotspot" designated in the state of West Bengal, by the Ministry of Health and Family Welfare (MOHFW), Government of India. Our hospital has a COVID working group since February 2020, which determines the hospital standard of practice in the management of COVID-19. Currently, we are performing in-house reverse transcription-polymerase chain reaction (RT-PCR) tests for SARS$\mathrm{CoV}-2$ and rapid respiratory virus detection panel.

The Indian health-care system has unique challenges in this pandemic with a large patient population, a relatively small number of health-care workers, and a shortage of essential equipment such as

\footnotetext{
This is an open access journal, and articles are distributed under the terms of the Creative Commons Attribution-NonCommercialShareAlike 4.0 License, which allows others to remix, tweak, and build upon the work non-commercially, as long as appropriate credit is given and the new creations are licensed under the identical terms.
}

For reprints contact: WKHLRPMedknow_reprints@wolterskluwer.com protective gear and testing kits. Limited resources in many centers do not allow setting up of separate hospital section for COVID patients. Cancer is a public health problem in India, with almost $8.3 \%$ of deaths being attributed to cancer. ${ }^{[1]}$ Therefore, continuing cancer care is critical to the Indian health system.

The purpose of this article is to describe the measures taken by the department of oncoradiology to control the infection and minimize staff exposure, both during the current lockdown period with reduced patient load and postlockdown period with increased demand for radiology services. We have also discussed algorithms and protocols unique to the practice of oncoradiology in the time of the COVID-19 pandemic.

\section{Special Consideration for Radiology}

Imaging is ordered in a RT-PCR-confirmed COVID-19 patient when there are moderateto-severe symptoms at diagnosis or there is progression of symptoms during the course of illness. Imaging may also be considered in patients with a high clinical suspicion of COVID-19 with moderate-to-severe symptoms and a negative RT-PCR. ${ }^{[2]}$

How to cite this article: Chatterjee $A$, Biswas BK, Gehani A, Das J, Sen S, Mukhopadhyay S, et al. Oncoradiology preparedness in the COVID-19 pandemic: Perspective from a tertiary oncology referral center from eastern India. Indian J Med Paediatr Oncol 2020;41:789-98.

\section{Argha Chatterjee ${ }^{1}$, Bivas K Biswas'2, Anisha Gehani ${ }^{1}$, Jayanta Das ${ }^{3}$, Saugata Sen ${ }^{1}$, Sumit Mukhopadhyay ${ }^{1}$, Aditi Chandra ${ }^{1}$, Priya Ghosh', Bharat Gupta ${ }^{1}$, Dayanand Lingegowda ${ }^{1}$}

${ }^{\prime}$ Department of Radiology and Imaging, Tata Medical Center, Kolkata, West Bengal, India, ${ }^{2}$ Department of Medical Oncology, Tata Medical Center, Kolkata, West Bengal, India, ${ }^{3}$ Department of Nuclear Medicine, Tata Medical Center, Kolkata, West Bengal, India

Submitted: 18 -May-2020 Revised: 31-Jul-2020 Accepted: 23-Aug-2020 Published: 18-Nov-2020

Address for correspondence: Dr. Argha Chatterjee, Tata Medical Center, 14 Main Arterial Road (E-W), New Town, Kolkata - 700 156,

West Bengal, India.

E-mail: arghachat84@gmail. com

Access this article online Website: www.ijmpo.org DOI: 10.4103/ijmpo.ijmpo_240_20 Quick Response Code:

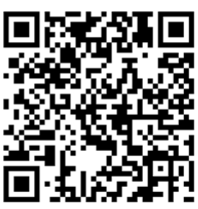


Because these investigations (portable radiography and high-resolution computed tomography of chest) require patient contact, it puts radiology staff at a high risk of infection. Fellows and consultants working in ultrasound and interventional radiology units are also susceptible to the high-risk exposure. An ultrasound examination is comparable to an outpatient examination, while interventional procedures may be comparable to a minor operative procedure.

Furthermore in the radiology department, a significant number of frontline workers are not physicians or nurses but radiology technologists, housekeeping staff, and health-care assistants, who do not have adequate training in infection control measures. We must delicately and proactively manage these vulnerable and valuable human resources.

\section{Transmission of SARS-CoV-2 Virus}

The presymptomatic stage of COVID-19 can last up to 14 days. The $\mathrm{WHO}$ has suggested that infection transmission may occur in the presymptomatic stage of the disease. ${ }^{[3]}$ The Centers for Disease Control and Prevention (CDC), USA, has suggested the implementation of source control measures for every person entering a health-care facility, regardless of their symptoms. ${ }^{[4]}$ Therefore, we have implemented source control measures for every patient and health-care worker with the policy that "anybody can be a potential source of infection."

COVID-19 spreads by respiratory droplets which usually do not travel beyond 1-2 $\mathrm{m}$ from the patient. There is no clinical evidence that suggests airborne spread in COVID-19 in the absence of aerosol-generating procedures (AGP). ${ }^{[5]}$ Therefore, the WHO continues to recommend droplet precaution in the absence of AGPs. ${ }^{[5]}$ Surgical three-ply masks offer enough protection against respiratory droplets $(5-10 \mu)$. N95/FFP3 masks are required in cases where an AGP is being performed. AGP is rare in diagnostic radiology, however, some interventional radiology procedures such as nasojejunal tube insertion are AGP.

Health-care professionals are particularly susceptible to infection. Health-care workers represented $4 \%$ of all cases in Wuhan and $13 \%$ of all cases in Spain. ${ }^{[6]}$ Health-care workers also transmit the disease to the community. A UK estimate on April 17, 2020, suggests that about one-third of cases are related to health-care workers. ${ }^{[7]}$ Health-care facilities can also be points of infection spread and source of onward transmission. A retrospective analysis from Wuhan, China, suggested that a hospitalassociated transmission was suspected in up to $41 \%$ of cases. ${ }^{[8]}$ Therefore, it is crucial to have a written protocol for radiology departments not only to protect radiology staff but also to limit disease spread in hospital settings.

\section{Triaging of Oncoradiology Services - Optimum Recommendation}

With the national lockdown in place, demand for the radiology services has reduced. However, we expect the demand for imaging to increase significantly once the lockdown is eased or lifted. A system of triage is necessary to provide quick services to those who need it the most while maintaining social distancing in the waiting area and departmental infection control practices. ${ }^{[9]}$

Radiological investigations are classified in three broad categories in oncology practice.

\section{Screening procedure for early detection}

Screening procedures that require clinical visits, such as mammography, can be postponed during this pandemic. To our knowledge, there is no recommendation from any professional body or society about the maximum permissible interval before screening can be resumed.

\section{Staging of newly diagnosed cancer patients}

Treatment may be postponed for any low-grade malignancy (low-grade sarcoma) or incidental findings or small tumor (such as subcentimeter renal tumor) without any aggressive biology. Hence, imaging for staging of these diseases may be put on hold..$^{[10]}$ Where immediate treatment is warranted, minimum imaging for primary tumor and symptom-directed screening for metastatic sites should be carried out. Invasive staging procedure, such as mediastinal staging in lung cancer, can be done during the time of definitive surgery if contemplated.

\section{Surveillance and follow-up imaging}

Surveillance and follow-up imaging should be deferred in patients with low risk of recurrence and where there is no effective subsequent line of treatment. The follow-up interval can be increased to the longest recommended frequency if feasible and telephonic consultation for follow-up is done. ${ }^{[10]}$

\section{General Department Planning}

\section{Screening of patients}

At the main reception, patients and their attendants are being screened for fever (with thermal scanning) and other symptoms of COVID-19 by trained nursing staff and health assistants. Both the patients and their relatives are screened, and masks and handrub are provided when patients do not have these in their possession. A history of international travel is taken. Suspected cases are being isolated in the isolation area at the emergency department and being screened by the on-call doctor of the respective subspecialty department. If their symptoms still cannot be explained by the primary neoplastic condition, these patients are being transferred to the isolation ward through a predetermined secure route. A swab is taken in the isolation room and sent 
for RT-PCR. All other patients are considered nonsuspect for COVID and they are sent to the respective subspecialty departments.

\section{General disinfection practices}

- The disinfection practices for different rooms and areas are summarized in Table 1

- Specific imaging protocols for suspected and known COVID-19 patients are described later

- All patients and patients' relatives should be wearing a mask of some form covering the nasal bridge to the chin as per the current government recommendation, however, a surgical mask is preferred. Respirators are discouraged

- The main door to the reception area is handled by a single security personnel.

\section{Crowd control}

- Entry to the hospital is restricted to one relative per patient

- The waiting area is rearranged to include fewer chairs so that there is an adequate physical distance between patients and the patients face away from each other

- The reception desk is cordoned off to maintain at least 1-m distance between the reception desk clerk and patient.

\section{Employee education and training}

Regular training and debriefing of employees are carried out in key areas:

- Proper use of appropriate personal protective equipment (PPE)

- Effective communication of problem areas within the department. Physical distancing is being encouraged for such communication, such as phone calls to replace inperson discussion as much as possible

- Proper respiratory etiquette (e.g., always cough or sneeze in a tissue or sleeve of shirt)

- Proper personal hygiene (e.g., men should be cleanshaven for proper fitting of mask)

- Not to carry personal belongings (laptops, large bags) if it can be avoided

- Mobile phone handling is to be kept at a minimum and should be done using a plastic cover while on duty

- Immediately after reaching home, soak and wash clothes in soapy water and take a bath

- Report any illness at the earliest.

Simple diagrams are distributed electronically among employees regarding infection control [Figure 1].

\section{Implementation of "Code Green"}

For a smooth transfer of patients, who are known or suspected to have COVID-19, we have implanted a new emergency announcement called "Code Green." Whenever such patients require transport to and from any

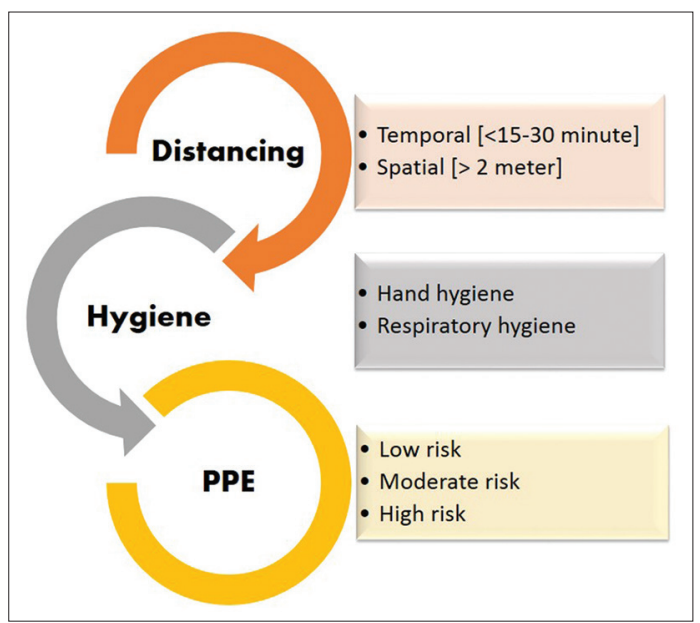

Figure 1: Standard infection control measures against coronavirus disease 2019. This simple chart helps in staff education and easy recall of basic steps of infection control

\begin{tabular}{lll}
\hline \multicolumn{2}{c}{ Table 1: General disinfection protocol } \\
\hline Area & Disinfectant & Frequency \\
\hline Patient table* & Bacillol 25 & After each patient \\
Floor and wall & 1\% sodium hypochlorite & 4 hourly \\
$\begin{array}{l}\text { Doorknobs/ } \\
\text { handles }\end{array}$ & $70 \%$ isopropyl alcohol & $\begin{array}{l}\text { As frequently as } \\
\text { possible }\end{array}$ \\
Ultrasound probe & Bacillol 25 & After each patient \\
X-ray detector & Bacillol 25 & After each patient \\
*We have stopped using linen on patient table (CT and ultrasound) \\
$\begin{array}{l}\text { and using a plastic sheet instead that is being discarded after each } \\
\text { patient in a closed lid container. CT - Computed tomography }\end{array}$
\end{tabular}

clinical department, including radiology, a "code green" is announced. This mobilizes the COVID transport team which includes housekeeping and security personnel who coordinate informing the primary referring physician, formation of a secure corridor for transfer, and sanitization of the points of contact after the transfer.

\section{General administration}

The department of radiology is proactively plugged into the COVID action group of the hospital. A daily critical review of literature is done and regular updates on hospital status are being shared through media platforms such as the departmental WhatsApp group. Daily walkabout and audits are conducted to ensure that infection control measures are properly and consistently practiced.

\section{Staggered rotation among employees}

Staggered rotation is implemented because of the following two reasons. ${ }^{[11]}$

- Restriction of staff exposure to avoid mass quarantine or sickness, which further limits workforce

- Limited in-house accommodation for staff in the time of severely restricted public transport during the lockdown.

Employees (consisting of radiologists, technologists, nurses, chaperones, reception desk assistants, and housekeeping) are 
divided into two to three groups depending on workforce. These groups are rotated so that each group can get 7 days of paid leave between rotations. We do not currently have staff members in the frontline who have a high risk of COVID-related illness; however, we recommend that such people should avoid moderate- to high-risk duties. Hospital buses are arranged for transport of staff during the lockdown period who cannot be accommodated or put in staggered rotation.

\section{Contact tracing}

After every new positive RT-PCR test is reported from our virology laboratory, the central command of the hospital implements active contact tracing among the staff. In radiology, contact tracing is carried out by reviewing the records for any radiology investigations performed on these patients within the past 14 days and each staff who is involved in such procedures is inquired about their symptoms and PPE use during that procedure.

\section{Quarantine policy}

The quarantine policy at our institute is as follows (for non-AGP only):

\section{High-risk exposure}

High-risk exposure is defined by contact $>15$ min within $1 \mathrm{~m}$ of the patient without a moderate- or high-risk PPE (minimum PPE to be worn for the patient encounter are N95 mask and face shield/goggles as per the institution policy). The staff will be quarantined for 14 days. The staff joins back on day 15 after an RT-PCR test comes negative on day 14 . Hydroxychloroquine prophylaxis is offered. ${ }^{[12]}$

\section{Low-risk exposure}

It is defined by patient contact within $1 \mathrm{~m}$ for $<15 \mathrm{~min}$ and without appropriate PPE. Symptoms are monitored without quarantine. Hydroxychloroquine prophylaxis is offered.

Any patient contact from a distance of $>1 \mathrm{~m}$ or while using recommended PPE is not considered an exposure and no further action is taken.

Staff who are positive for SARS-CoV-2 are treated according to the current government guidelines.

\section{Educational activities}

- All departmental seminars are conducted over webbased video collaboration platforms (Microsoft Teams, Google Meet, etc.)

- All students and observers are sent on leave for the time being.

\section{Risk Estimation and Appropriate Personal Protective Equipment [Figure 2]}

We have divided our staff into three risk strata based on their risk of significant contact with the SARS-CoV-2positive patient. Significant contact is defined as a person

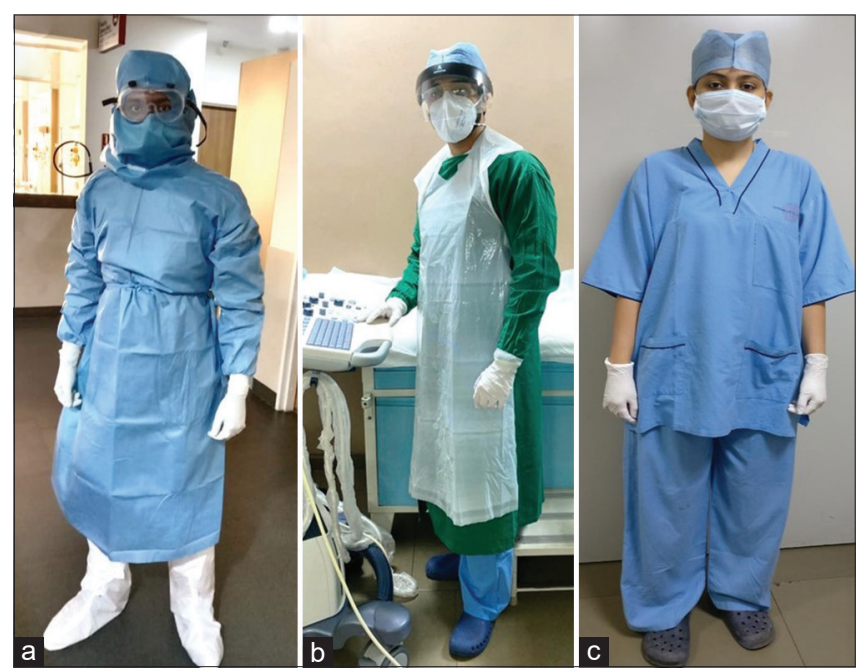

Figure 2: Personal protective equipment. (a) High-risk personal protective equipment. (b) Moderate-risk personal protective equipment. (c) Low-risk personal protective equipment. N95 mask is not mandatory in moderaterisk and low-risk personal protective equipment (except in prolonged procedures in moderate-risk areas)

spending $>15$ min within $1 \mathrm{~m}$ of a suspected or confirmed COVID patient without adequate personal protection. ${ }^{[3]}$ Risk stratification helps in the judicial use of PPE. People working in different risk areas should not be intermingling as much as possible.

\section{High-risk areas}

1. Radiographers performing portable radiography in an isolation ward

2. Doctors performing portable ultrasound in an isolation ward

3. Doctors performing image-guided procedures in an isolation ward or in a suspected/confirmed COVID patient

4. Nurses attending image-guided procedures in an isolation ward or in a suspected/confirmed COVID patient.

We are not performing computed tomography $(\mathrm{CT})$ in known or suspected COVID patients routinely. If CT is performed in such cases, radiographers are not entering the gantry, and patients are being positioned either by themselves or by the accompanying isolation ward staff. The CT table is adjusted remotely from the console afterwards.

Recommended PPE for the high-risk areas consist of:

- Scrubs are mandatory

- At least $70 \mathrm{~g} / \mathrm{m}^{2}$, unwoven, coverall, disposable full-sleeved gown

- Disposable headcover

- Visor or face shield

- Disposable double surgical gloves

- N95 or FFP-2 mask. We are not using respirators

- Disposable shoe cover. There is emerging evidence that a shoe cover is not necessary. ${ }^{[13]}$ However, we are continuing its use in line with the hospital policy. 
Proper donning and doffing procedures are being followed under supervision.

\section{Moderate-risk area}

1. Doctors performing ultrasound in general patients

2. Radiographers positioning general patients and performing barium procedures

3. CT, magnetic resonance imaging (MRI), and positron emission tomography (PET)-CT technologists working inside the gantry in general patients

4. CT surveillance radiologist

5. Nurses, housekeeping, and chaperones working in ultrasound, CT, and MRI gantry

6. Housekeeping who are transporting patients and posted at moderate-risk area.

Recommended PPE for the moderate-risk areas consist of:

- Scrubs/uniforms are mandatory

- Full-sleeve gown (for radiographers and sonologists)

- Disposable plastic apron covering the neck to knee

- Disposable surgical cap

- Visor or face shield, being reused after cleaning with soap water and alcohol

- Disposable double surgical gloves. Outer gloves are changed or sanitized between patients

- N95 or FFP-2 masks. Instead of an N95 mask, a surgical mask may be acceptable.

\section{Low-risk areas}

1. Radiologists reporting from a separate workstation

2. CT/MR technologists working inside the console room

3. Reception desk clerks

4. Housekeeping who are posted outside a moderate-risk area.

Recommended PPE:

- Disposable surgical cap

- Single surgical gloves. Gloves are changed or sanitized as per standard protocol

- Surgical masks. Personal preference to use N95 or FFP-2 masks is not being discouraged.

The MOHFW has recommended low-risk PPE for general radiology practice. ${ }^{[14]}$ However, considering the variable risk of exposure within the department, we have escalated the PPE requirement accordingly.

\section{Optimal use of N95 mask}

Use of N95/FFP2 mask is mandatory in two areas:

- Inside a COVID isolation ward

- AGP or prolonged procedures (such as ultrasound-guided biopsy and fine-needle aspiration cytology) in interventional radiology.

Standard three-layered surgical masks with laces (not elastic string) may be sufficient in all other areas. Routine use of N95 mask has two drawbacks:

- Prolonged use of N95 masks (>4-6 h) with full aseptic precaution is not practical as it causes extreme discomfort to the wearer. ${ }^{[15,16]}$ We are, therefore, using it for short periods and short shifts. We understand that most radiology departments in India do not have the workforce to rotate the staff in 4-h shifts, especially with a staggered rotation in place. This makes routine use of N95 masks difficult

- N95 masks are in short supply. They are currently being reused as per protocols described by the CDC and All India Institute of Medical Sciences. Each wearer has been provided four sets of masks and they are asked to rotate the masks from day 1 to day 4 . In this way, the mask worn on day 1 is getting a "drying-off" time of $72 \mathrm{~h}$ before being reused on day 5 .

Furthermore, there is evidence that in routine outpatient and inpatient care, an N95 mask offers no extra protection to a viral infection such as influenza compared to a surgical three-ply mask, ${ }^{[17,18]}$ especially if they are not fit tested. To our knowledge, most institutes in India are not carrying out routine fit tests for N95 masks as there are limited resources to do so.

\section{Donning and doffing procedure}

A detailed description of donning and doffing of high-risk PPE is beyond the scope of our article. However, donning and doffing of the moderate- and low-risk PPE should also be done properly. A basic algorithm to remember is wash-body-face-eye-hand. Before donning the PPE, hand wash should be done for at least $20 \mathrm{~s}$ followed by wearing the inner gloves and gown. Next, the mask and goggles are worn in that order. Finally, the outer gloves are worn over the sleeve of the gown. The doffing is done in the reverse order. ${ }^{[19]}$ Hand washing is done at the end of the doffing procedure.

\section{General Radiography and Mammography}

- Radiographers must wear moderate-risk PPE and patients must wear a disposable surgical mask

- Portable radiography for non-COVID wards is being considered a moderate-risk procedure. We mandate an N95/FFP2 mask in such situations as patients often have symptoms such as cough that may result in significant exposure. During portable radiography in the general intensive care unit (ICU), an additional nondisposable surgical gown is being provided to the radiographer as per our ICU policy. Standard precautions are being followed and routine machine cleaning is being performed.

\section{General Computed Tomography}

CT is the workhorse of most oncoradiology departments and cannot be scaled down. Standard precautions need to be followed during the CT scan of every patient.

\section{Minimal gantry work}

Time spent inside the gantry should be kept to a minimum. Staff who are not wearing a moderate-risk PPE should not be entering the CT gantry. 


\section{Radiologist for surveillance}

One radiologist is constantly posted within the CT console who wears a moderate-risk PPE. The duties of this person include:

- Active monitoring of thoracic CT for features for COVID and early notification

- Examination of patients who develop contrast reactions or any other clinical issue

- The first response in case of a code blue.

We are in the process of obtaining a virtual console setup from Siemens (R), Erlangen, Germany (Virtual cockpit) that will enable consultant radiologists to perform remote surveillance in real time, even during night shifts.

\section{General Ultrasound}

\section{Fever ultrasound}

Patients who have some suspicious symptoms but are not eligible to be isolated as per hospital policy are still examined with extra precaution in a separate room designated "fever ultrasound." Other than wearing a moderate-risk PPE, nurses and performing radiologists do not leave this room frequently.

\section{Routine ultrasound}

In all other cases, the doctor and nurse wear moderate-risk PPE and use a fresh pair of clean gloves for each patient. The examination must be optimal and brief. The use of probe cover for each patient, although desirable, is not cost-effective.

\section{General Magnetic Resonance Imaging}

In oncology, most MRI studies in a confirmed or suspected COVID patient can be deferred or replaced by CT. In case of a possible stroke in a COVID patient, we perform a CT head with perfusion, as necessary.

Metal strips over the nasal bridge in some masks may not be MRI compatible and should be changed to a mask with a plastic strip before entering the MRI scanner. Tape may be applied across the bridge of the nose to maintain a tight seal.

\section{General Nuclear Medicine}

\section{Limitation of resources and deferral of services}

- Due to the closure of international borders, we cannot obtain ${ }^{99} \mathrm{~m}$ technetium and ${ }^{131}$ iodine-related radiopharmaceuticals. Therefore, major diagnostic procedures such as radio-iodine scans or bone scans are not being performed. Radio-iodine ablation therapy has been suspended since the last week of March

- We have a limited supply of18fluorodeoxyglucose (18F-FDG) from local cyclotron operator, therefore FDG-PET scan is scaled down to 3 days a week. ${ }^{68} \mathrm{Ga}$-prostate-specific membrane antigen-PET and
${ }^{68} \mathrm{Ga}$-DOTA-PET are being performed, as we have our institutional gallium generator.

\section{Infection control measures}

- Because all nuclear medicine scans require a prolonged stay of the patient within the scanner and holding area, thorough cleaning of the waiting area is done with $1 \%$ sodium hypochlorite solution in addition to standard procedures mentioned in Table 1 .

\section{Radiography for Confirmed/Suspected Coronavirus Disease Patients in Isolation Ward}

\section{Indication}

A portable chest radiograph is the initial imaging of choice in a confirmed and symptomatic COVID patient.

- As baseline imaging. The Fleischner Society recommends that no imaging is needed in the presence of mild symptoms unless there is a risk of progression. ${ }^{[20]}$ However, in an oncology demographic such as ours, we consider every patient to be at high risk of progression

- To rule out alternative diagnoses such as lobar consolidation, pleural effusion, or heart failure

- Comparison with previous imaging. A new finding in a known patient may explain the clinical features and unnecessary isolation may be avoided.

\section{Logistics}

One portable computed radiography (CR) machine is designated for the COVID-19 isolation ward. Our isolation ward consists of six negative-pressure ventilation rooms. The designated machine is stored outside the rooms within the ward which is in the second building (henceforth phase 2) in our campus. We are performing radiographs in two shifts and each shift is covered by two technologists. No technologist is posted within the ward. We have designated two cassettes for COVID patients to restrict contamination.

\section{Technique}

Transportation of cassette to and from the image reader is being done with full aseptic precaution. The cassette is first cleaned with $70 \%$ isopropyl alcohol. Then, the cassette is put inside a clean plastic (yellow garbage bag) bag and a water-tight seal is obtained with a surgical tape. The cassette is then taken to the isolation ward where it is put inside a second plastic bag and sealed similarly. Radiographers carrying the cassette are wearing moderate-risk PPE during transport. Before entering the isolation room, one of the radiographers washes hands, dons the high-risk PPE, and takes the machine inside the isolation room with the double-wrapped cassette. After the exposure, this person transfers the cassette aseptically to the other radiographer in the antechamber after opening the outer plastic cover. Then, it is immediately cleaned with alcohol. The first radiographer brings the machine out 
in the antechamber and thoroughly cleans it and doffs the PPE. The PPE is discarded safely in a closed bin.

During the entire process, the patient needs to be wearing an N95/FFP2 mask unless being ventilated.

\section{Computed Tomography for Confirmed/ Suspected Coronavirus Disease Patients in the Isolation Ward}

Department policy for computed tomography in coronavirus disease

- CT is not used in the screening or diagnosis of COVID-19. ${ }^{[21,22]}$ A negative CT provides false reassurance and CT features described in COVID-19 are nonspecific. We understand in certain high-risk regions in India that routine CT screening of preoperative or indoor patients is being undertaken to limit transmission among health-care workers. We did not find any evidence to support this practice. The American College of Radiology and the Society of Thoracic Radiology and American Society of Emergency Radiology have all advised against this practice in their position statements

- Routine use of chest CT is not indicated even in symptomatic RT-PCR-positive patients as the CT feature does not necessarily correlate with clinical course. CT is done when there is a sudden or unexplained change in clinical course or worsening of respiratory symptoms. ${ }^{[20]}$ In such cases, a noncontrast high-resolution chest $\mathrm{CT}$ is necessary and sufficient

- CT pulmonary angiography in COVID patients should be limited considering the low specificity of raised D-dimer level in COVID patients and the requirement of high-flow contrast dose.

\section{Patient movement}

- CT scanner in Phase 2 building is designated for COVID-19 patients

- A designated route is used for the transfer of confirmed COVID-19 patients from the isolation ward to the CT room so that the route of the patient should not cross the route of technologists and radiologists [Figure 3]

- The technologist will not enter the gantry without a high-risk PPE. The patient will be positioned by the attendant and the CT table will be remotely adjusted

- During the entire process, the patient needs to be wearing an N95/FFP2 mask unless ventilated.

\section{Air-conditioning issues}

- There are concerns regarding the spread of the virus through the vents of the centralized air-conditioning (AC) system. However, because the patient is wearing a mask or is in a closed ventilation circuit, the risk of spread is minimal. Furthermore, there is only limited laboratory evidence of airborne spread of SARS-CoV-2 ${ }^{[5]}$

- If any CT unit has an isolated air-handling unit (AHU), it may be advisable to switch off the $\mathrm{AC}$ for the duration of the scan followed by a 60 -min downtime. During this period, a backup split AC may be used, if available

- Our department has a common AHU for CT and MRI scanner; therefore, we are not switching the AC off during the procedure to protect the MR scanner.

\section{Postprocedure cleaning of surfaces}

- Cleaning of all surfaces is done after the scan in top-to-bottom, clockwise, and linear fashion using 1\% sodium hypochlorite followed by a 60 -min downtime before the next patient can be taken.

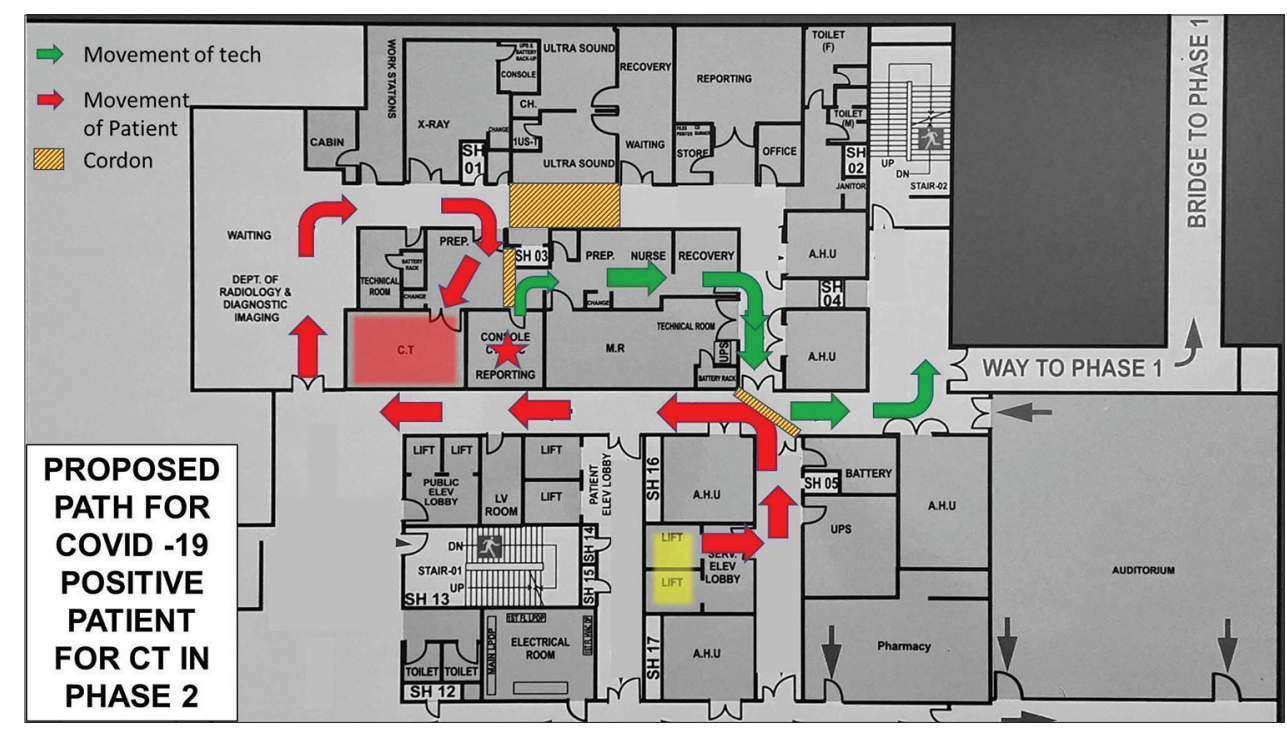

Figure 3: Plan for safe transport of known or suspected coronavirus disease 2019 patients from isolation ward. Small area painted light yellow is the elevator earmarked for patient movement from the isolation ward to the first floor computed tomography. Area painted light red is the computed tomography gantry. The red arrow marks the pathway of the patient and her attendants. The green arrows marks the pathway of the technologists coming in and out of the computed tomography console (asterisk). Cross-hatched yellow bars represent the cordon between these two pathways to avoid exposure of technologists who will be wearing moderate-risk personal protective equipment. The technologist will not enter the computed tomography gantry and will remotely adjust the computed tomography table 


\section{Interventional Radiology}

Special consideration is needed for interventional radiology for two reasons:

- Prolonged contact with patients is expected for most procedures ( $>15 \mathrm{~min})$

- This is the only section in radiology where AGP is conducted.

Aerosol-generating procedure ${ }^{[23]}$

- Nasogastric or nasojejunal tube insertion

- Lung biopsy or any procedure on the lung, when complicated by hemoptysis or violent coughing. However, lung biopsy is not an AGP per se.

\section{Patient selection}

Before elective procedures, we are now performing RT-PCR for SARS-CoV-2 along with other routine serology. In case of emergency procedures that are prolonged, a swab is being sent for RT-PCR and high-risk precautions are being taken for the procedure. This is in accordance to the circular released by the Directorate of Health Services, Government of West Bengal, on April 28, 2020. ${ }^{[24]}$

\section{Personal protective equipment}

In interventional AGP procedures, an N95 mask is being used with a moderate-risk PPE. For all procedures, the patient must wear a surgical mask, and in case of lung biopsy, the patient must wear an N95 mask. Oxygendependent patients may wear a nonrebreathing mask, or a surgical mask may be worn under the oxygen mask.

\section{Active Surveillance of Chest Radiograph and Chest Computed Tomography}

Even though CT screening of COVID-19 is not recommended, anecdotal evidence suggests that incidental detection of COVID-19 on CT scan or chest radiograph is

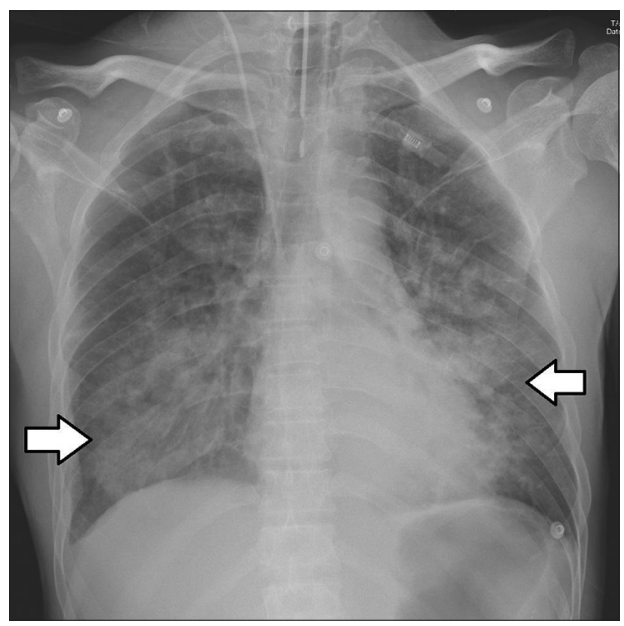

Figure 4: Chest radiograph, postero-anterior view, of a coronavirus disease 2019 patient with acute lymphoblastic leukemia. Bilateral focal consolidations are noted (arrows) possible. Therefore, all thoracic $\mathrm{CT}$ and chest radiographs are being monitored in real time. In this way, concerned patients can be isolated and tested at the earliest.

Policy

- Any new unilateral or bilateral ground-glass opacity in the lungs is notified to the attending radiologist

- A review of previous CT, underlying malignancy, and ongoing treatment (especially pneumonotoxic chemotherapy) is conducted

- If suspicion of COVID-19 persists, the primary physician is notified immediately along with the microbiology action team and hospital administration. CT room is thoroughly disinfected with a downtime of $60 \mathrm{~min}$.

The following are the high-risk features listed in our department guidelines.

\section{Chest radiograph [Figure 4]}

- New peripheral, bilateral, ground-glass opacities and/or consolidations.

\section{Chest computed tomography [Figure 5]}

- New multifocal, peripheral, subpleural, and bilateral ground-glass opacities

- Subpleural sparing may be present

- Ground-glass opacities can be rounded or not sharply demarcated.

- New focal or bilateral crazy paving or consolidations or organizing pneumonia pattern.

Features that should indicate other pathologies

- Lymphadenopathy

- Cavitation

- Tree-in-bud/centrilobular nodular distribution

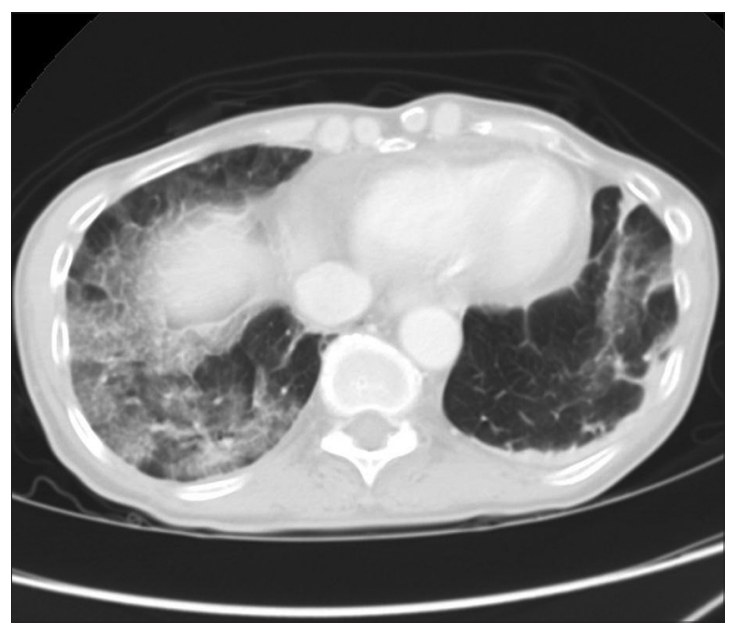

Figure 5: Computed tomography scan of a case of incidentally detected coronavirus disease 2019. Known case of lung malignancy underwent upper abdominal computed tomography for staging workup. Computed tomography sections in the lung window through lung bases show focal ground peripheral glass densities bilaterally, right more than left 


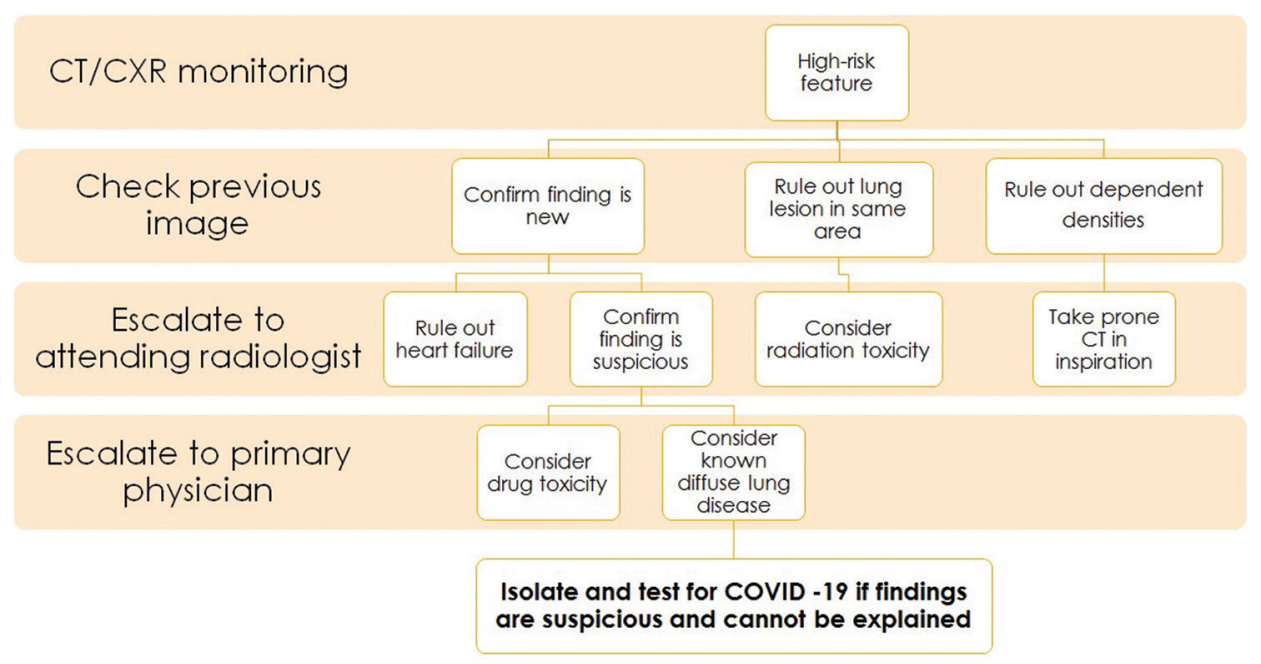

Figure 6: Simple algorithm for imaging surveillance and notification of coronavirus disease 2019 in the radiology department

- Mass-like lesions

- Lobar consolidation. ${ }^{[25]}$

Pleural effusion is an uncommon feature of COVID-19 pneumonia; however, it may be found commonly in oncology patients due to an underlying liver or renal disease, hypoproteinemia, or tumor deposits. Therefore, it should not be used as a determining feature.

\section{Common confounding condition in oncology practice}

The above-mentioned features are not specific for COVID-19 and may occur in multiple conditions including other viral pneumonia. Any new densities that may represent infection should be investigated. However, some conditions that mimic COVID-19 can be excluded by associated radiological and clinical findings and may not be escalated further. Radiologists need to be aware of these conditions and should be communicated accordingly.

- Dependent densities due to inadequate inspiration while performing CT

- Cardiogenic pulmonary edema

- Chemotherapy- and immunotherapy-related toxicities (most common offending drugs are given below)

- Bleomycin

- Paclitaxel/docetaxel

- Gemcitabine

- Erlotinib

- Nivolumab.

- Radiation toxicity

- Posttreatment focal fibrosis in a lung mass.

A short algorithm is being used by our trainee radiologists [Figure 6]. This algorithm is not exhaustive, and each case needs to be considered separately. To err on the side of caution, any suspected patient should undergo RTPCR.

\section{Conclusion}

COVID-19 is an unprecedented challenge posed to the imaging practice. Knowledge of this new disease is still emerging, and all recommendations and protocols should be kept as fluid as possible. We hope by following a standard protocol, oncoradiology departments should be able to function smoothly without restricting patient care.

Financial support and sponsorship

Nil.

\section{Conflicts of interest}

There are no conflicts of interest.

\section{References}

1. India State-Level Disease Burden Initiative Cancer Collaborators. The burden of cancers and their variations across the states of India: The Global Burden of Disease Study 1990-2016. Lancet Oncol 2018;19:1289-306.

2. Jajodia A, Ebner L, Heidinger B, Chaturvedi A, Prosch H. Imaging in corona virus disease 2019 (COVID-19)-A Scoping review. Eur J Radiol Open 2020;7:100237. Published 2020 May 11. doi:10.1016/j.ejro.2020.100237.

3. World Health Organization. Coronavirus Disease 2019 (COVID-19): Situation Report - 73. Geneva: World Health Organization; 2000. Available from: https://www.who.int/docs/ default-source/coronaviruse/situation-reports/20200402-sitrep-73covid-19.pdf. 20. [Last accessed on 2020 May 10].

4. Centers for Disease Control and Prevention. 2020. Coronavirus Disease 2019 (COVID-19). Available from: https://www.cdc.gov/ coronavirus/2019-ncov/hcp/infection-control.html. [Last accessed on 2020 Aug 11].

5. World Health Organization. Modes of transmission of Virus Causing COVID-19: Implications for IPC Precaution Recommendations. Scientific Brief, $9^{\text {th }}$ of July 2020. Geneva: World Health Organization; 2020. Available from: https://www. who.int/newsroom/commentaries/detail/modes-of-transmissionof-virus-causing-covid-19-implications-for-ipc-precautionrecommendations. [Last accessed 2020 Aug 11]. 
6. Parra A, Rising D. Spain's coronavirus death toll surpasses China as world struggles with containment. Global News 2020. Available from: https://globalnews.ca/news/6729174/coronavirusspain-death-toll-china/. [Last accessed on 2020 May 12].

7. Heneghan C, Oke J, Jefferson T. COVID-19: How Many Healthcare Workers are Infected? Oxford: The Centre for Evidence-Based Medicine; 2020. Available from: https://www. cebm.net/covid-19/covid-19-how-many-healthcare-workers-areinfected/html. [Last accessed on 2020 Apr 17].

8. Wang $\mathrm{D}, \mathrm{Hu} \mathrm{B}, \mathrm{Hu} \mathrm{C}, \mathrm{Zu} \mathrm{F}$, Liu X, Zhang J, et al. Clinical characteristics of 138 hospitalized patients with 2019 novel coronavirus-infected pneumonia in Wuhan, China. JAMA 2020;323:1061-9.

9. Mossa-Basha M, Meltzer CC, Kim DC, Tuite MJ, Kolli KP, Tan BS. Radiology department preparedness for COVID-19: Radiol Sci Expert Rev Panel 2020;296:E106-12.

10. ASCO. Cancer Screening, Diagnosis, Staging \& Surveillance. Available from: https://www.asco.org/asco-coronavirus-resources/ care-individuals-cancer-during-covid-19/cancer-screeningdiagnosis-staging. [Last accessed on 2020 Aug 11].

11. Mukherjee P, Lim TC, Chawla A, Chou H, Peh WC. Adaptability and responsiveness: Keys to operational measures in a regional hospital radiology department during the current COVID-19 pandemic. BJR Open 2020;2:20200017.

12. Ministry of Health and Family Welfare, India. Advisory on the Use of Hydroxychloroquine as Prophylaxis for SARS-CoV-2 Infection. Ministry of Health and Family Welfare. New Delhi: Government of India; 2020. Available from: https://www.mohfw. gov.in/pdf/AdvisoryontheuseofHydroxychloroquinasprophylaxi sforSARSCoV2infection.pdf. [Last accessed on 2020 May 10].

13. Crocker AD, Pipili E, Sturdee DW, Wilson KA. An investigation into the mechanism of the menopausal hot flush [proceedings]. J Pharm Pharmacol 1978;30 Suppl:59P.

14. Ministry of Health and Family Welfare, India. Novel Coronavirus Disease 2019 (COVID-19): Additional Guidelines on Rational use of Personal Protective Equipment (setting approach for Health Functionaries Working in Non-COVID areas). New Delhi: Ministry of Health and Family Welfare Government of India; 2020. Available from: https://www.mohfw.gov.in/ pdf/AdditionalguidelinesonrationaluseofPersonalProtectiveEqu ipmentsettingapproach forHealthfunctionarieswor kinginnonCOVIDareas.pdf. [Last accessed on 2020 May 09].

15. Li Y, Tokura H, Guo YP, Wong AS, Wong T, Chung J, et al. Effects of wearing N95 and surgical facemasks on heart rate, thermal stress and subjective sensations. Int Arch Occup Environ Health 2005;78:501-9.

16. Sinkule EJ, Powell JB, Goss FL. Evaluation of N95 respirator use with a surgical mask cover: Effects on breathing resistance and inhaled carbon dioxide. Ann Occup Hyg 2013;57:384-98.
17. Long $\mathrm{Y}, \mathrm{Hu} \mathrm{T}$, Liu $\mathrm{L}$, Chen $\mathrm{R}$, Guo $\mathrm{Q}$, Yang $\mathrm{L}$, et al. Effectiveness of N95 respirators versus surgical masks against influenza: A systematic review and meta-analysis. J Evid Based Med. 2020;13(2):93-101.

18. Radonovich LJ Jr, Simberkoff MS, Bessesen MT, Brown AC, Cummings DAT, Gaydos CA, et al. N95 respirators vs medical masks for preventing influenza among health care personnel: A randomized clinical trial. JAMA 2019;322:824-33.

19. European Centre for Disease Prevention and Control. Guidance for Wearing and Removing Personal Protective Equipment in Healthcare Settings for the Care of Patients with Suspected or Confirmed COVID-19. European Centre for Disease Prevention and Control; 2020. Available from: https://www.ecdc.europa.eu/ en/publications-data/guidance-wearing-and-removing-personalprotective-equipment-healthcare-settings. [Last accessed on 2020 May 13].

20. Rubin GD, Ryerson CJ, Haramati LB, Sverzellati N, Kanne JP, Raoof S, et al. The role of chest imaging in patient management during the COVID-19 pandemic: A multinational consensus statement from the Fleischner society. Chest 2020;158:106-16.

21. American College of Radiology. ACR Recommendations for the use of Chest Radiography and Computed Tomography (CT) for Suspected COVID-19 Infection. American College of Radiology; 2020. Available from: https://www.acr.org/Advocacyand-Economics/ACR-Position-Statements/Recommendations-forChest-Radiography-and-CT-for-Suspected-COVID19-Infection. [Last accessed on 2020 May 13].

22. Revel MP, Parkar AP, Prosch H, Silva M, Sverzellati N, Gleeson F, et al. COVID-19 patients and the radiology department Advice from the European Society of Radiology (ESR) and the European Society of Thoracic Imaging (ESTI). Eur Radiol 2020;30:4903-9.

23. Tan BS, Tay KH, Tan BH, Chung JW, Gangi A, Binkert C, et al. Joint CIRSE-APSCVIR checklist to prepare IR departments for COVID 19. Cardiovascular and Interventional Radiological Society of Europe; 2020. https://www.cirse.org/education/covid19-resource-centre/html. [Last accessed on 2020 May 12].

24. Directorate of Health Services, Government of West Bengal. Circular no. HPH/9M-21/2020/99. Directorate of Health Services. Kolkata: Government of West Bengal; 2020. Available from: https://www.wbhealth.gov.in/uploaded_files/corona/ CIRCULAR.pdf. [Last accessed 2020 May 05].

25. Simpson S, Kay FU, Abbara S, Bhalla S, Chung JH, Chung M, et al. Radiological Society of North America Expert Consensus Statement on Reporting Chest CT Findings Related to COVID-19. Endorsed by the Society of Thoracic Radiology, the American College of Radiology, and RSNA - Secondary Publication. J Thorac Imaging 2020;35:219-27. 